\title{
Using light-sheet fluorescence microscopy to identify anatomical structures and infection by infectious laryngotracheitis virus in chicken tissues
}

Matthew Smallridge $^{1}$, Mauricio J. C. Coppo ${ }^{1}$, Gabriela Segal ${ }^{2}$, Ellie Cho $^{2}$, Carol A. Hartley ${ }^{1}$

${ }^{1}$ Asia Pacific Centre for Animal Health, Faculty of Agricultural and Veterinary Science, The University of Melbourne, Australia.

${ }^{2}$ Biological optical microscopy platform, The University of Melbourne, Australia

\section{Abstract Text}

Infectious laryngotracheitis virus (ILTV) is an alphaherpesvirus that causes respiratory disease in infected chickens. First documented in Australia in 1935, ILTV has become endemic in throughout the country, with recombination resulting in the formation of new, more virulent strains. Due to its infectious nature and high morbidity rates, ILTV has become a focus for both industry and research. This study aims to investigate the applicability of light-sheet fluorescence microscopy for identification of anatomical structures and the detection of ILTV infection in chicken embryos or chicks.

Specific pathogen free white leghorn embryonated eggs or chicks were used in this study. Fourteen-day old embryos and 4-day old chicks were inoculated with ILTV that produced either mCherry or green fluorescent protein (GFP) during infection. All chicks and embryos were euthanised four days post inoculation and dissected into cranial, neck-lower beak, and three thoracic sections. The legs, wings and lower body sections were not investigated. The EyeCi method described by Henning et al. (2019) was used for full body clearing of dissected chicken and embryo sections. Due to the 
requirement of bleaching, tissues were permeabilised, and probed with mCherry and GFP targeted fluorescence booster antibodies labelled with AlexaFluor 647 prior to dehydration and clearing with ethyl-cinnamate. Images were acquired using a LaVsion

Ultramicroscope II Light Sheet Microscope, with sections captured as mosaics with a with $5 \mu \mathrm{m}$ z-step, and the microscopes minimum magnification of 1.26. All image stitching and rendering was conducted using Imaris software.

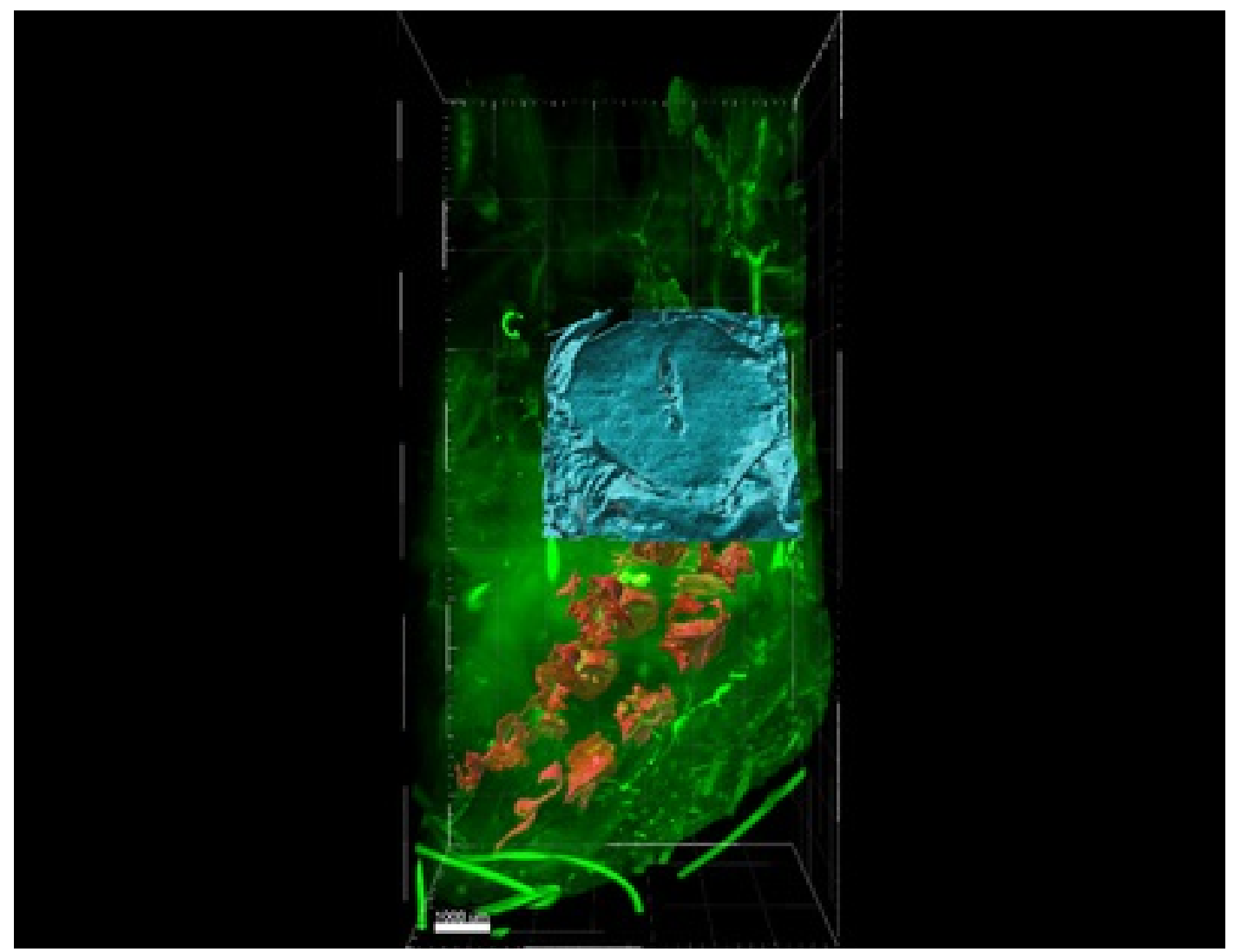

Figure 1: ILTV-GFP infected neck-lower beak section with surfaced larynx (blue) and vertebrae (red).

Clearing was effective in embryos, however insufficient clearing in chick tissues for accurate imaging to occur. Embryo tissues produced clear images, and anatomical structures of the upper respiratory tract were identified and rendered using Imaris. The cranial thoracic section provided the clearest images for both the mCherry-boosted ILTV-mCherry infected and uninfected control. To allow for individual 
analysis, structures of interest were cropped into smaller regions for rendering. The crop and trachea were surfaced and analysed, although no significant difference in fluorescence intensity was recorded.

ILTV-GFP infected neck-lower beak sections produced clear images with vertebrae and larynx clearly identified and surfaced (Figure 1). The ILTV-mCherry infected, and uninfected section had a smaller area imaged, and the hyoid apparatus was clearly identified in the ILTV-mCherry infected sample. The hyoid apparatus (HA) was also identified in the uninfected sample, with an obvious difference in fluorescence intensity of the HA between it and the infected section (Figure 2).

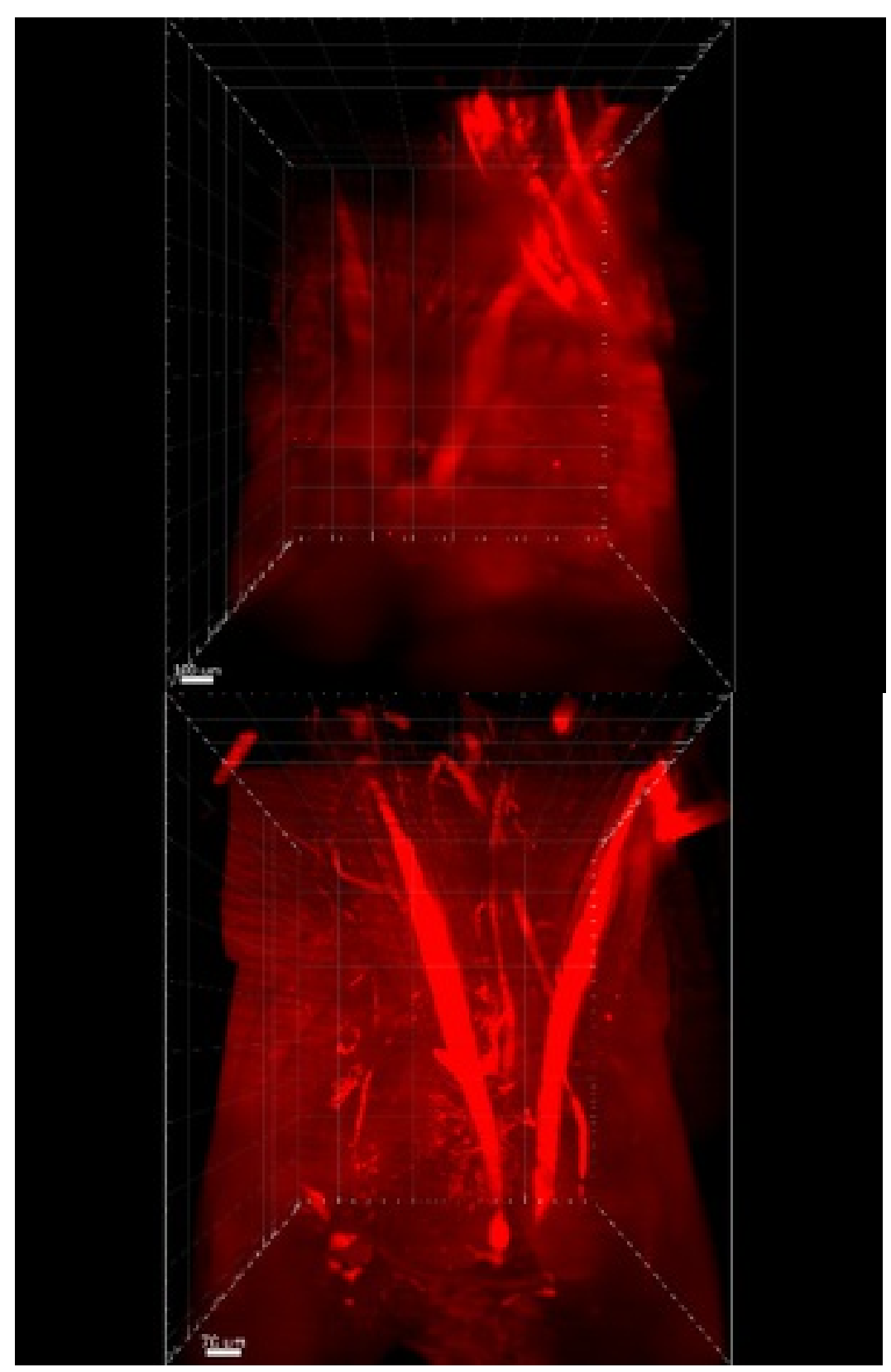


Figure 2: ILTV-mCherry infected (right) and uninfected (left) necklower beak section fluorescent images with visible HA

In conclusion, this study shows that LSFM is a viable technique to investigate the anatomy of chickens with evidence to suggest that identification of infected tissue is possible. The EyeCi clearing technique was an effective to clear embryonic tissue for imaging, however, further work is required to optimise the method for chick tissues. Further study is needed to determine if LSFM is a viable analysis method for virus infection in ovo and in vivo. 